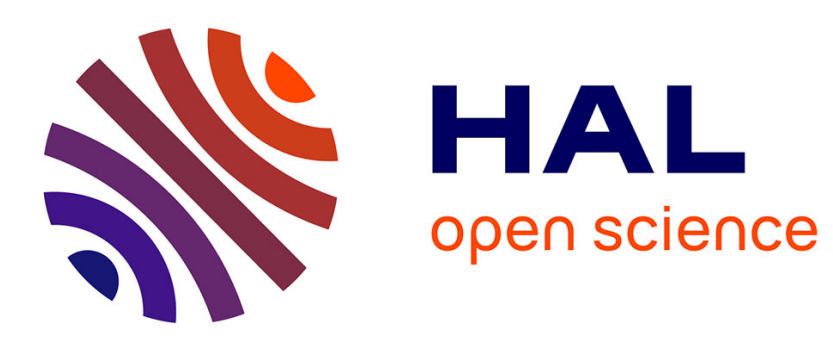

\title{
Occurrence of 2-methyl-, 3-methyl- and 6-methyltriaromatic steroid hydrocarbons in geological samples
}

Eric Lichtfouse, Joseph Riolo, Pierre Albrecht

\section{- To cite this version:}

Eric Lichtfouse, Joseph Riolo, Pierre Albrecht. Occurrence of 2-methyl-, 3-methyl- and 6methyltriaromatic steroid hydrocarbons in geological samples. Tetrahedron Letters, 1990, 31 (27), pp.3937-3940. 10.1016/S0040-4039(00)97511-7 . hal-00175050

\section{HAL Id: hal-00175050 https://hal.science/hal-00175050}

Submitted on 26 Nov 2007

HAL is a multi-disciplinary open access archive for the deposit and dissemination of scientific research documents, whether they are published or not. The documents may come from teaching and research institutions in France or abroad, or from public or private research centers.
L'archive ouverte pluridisciplinaire HAL, est destinée au dépôt et à la diffusion de documents scientifiques de niveau recherche, publiés ou non, émanant des établissements d'enseignement et de recherche français ou étrangers, des laboratoires publics ou privés. 


\title{
OCCURRENCE OF 2-METHYL-, 3-METHYL- AND 6-METHYLTRIAROMATIC STEROID HYDROCARBONS IN GEOLOGICAL SAMPLES.
}

\author{
E. LICHTFOUSE, J. RIOLO and P. ALBRECHT*
}

Département de Chimie, Université Louis Pasteur, 1 , rue Blaise Pascal, 67008 Strasbourg, France.

\begin{abstract}
Triaromatic steroid hydrocarbons bearing a methyl substituent at position 2, 3, or 6 have been identified in marine sediments and petroleums from the Paris basin by comparison with $C_{21}(1,2,3), C_{27}(4)$ and $\mathrm{C}_{29}$ (5) synthetic standards. Due to their formation with depth they are useful for maturity studies.

Molecular organic geochemistry has been increasingly used to understand the origin and transformation of organic matter in the subsurface. Petroleums and rock extracts contain a wide variety of molecules, called "biological markers", the structures of which suggest a link with biological substances. Sedimentary steroids 1 are inherited from sterols of living organisms at the time of deposition. Their structural variety (i.e. steranes, sterenes, aromatic steroid hydrocarbor:s) gives evidence for geochemical transformations undergone by their precursors due to factors such as microbial alteration, time, temperature and mineral catalysis.
\end{abstract}

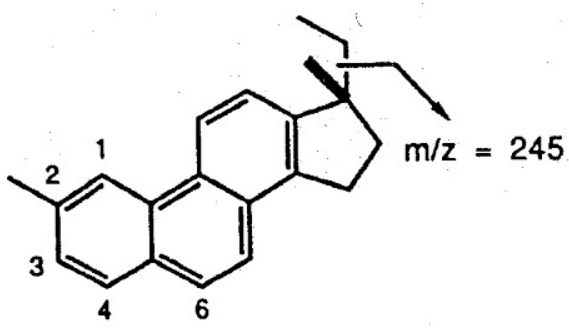

1<smiles>CC[C@]1(C)CCc2c1ccc1c2cc(C)c2ccccc21</smiles>

3<smiles>CC[C@]1(C)CCc2c1ccc1c2ccc2cc(C)ccc21</smiles>

2<smiles>[R]C(CC[C@@H](C)[C@]1(C)CCc2c1ccc1c2ccc2cc(C)ccc21)C(C)C</smiles>

$$
\begin{aligned}
& \text { 4: } R=H \\
& 5: R=C_{2} H_{5}
\end{aligned}
$$


The 4-methyl steroid derivatives found in ancient sediments and crude oils2,3 have for a long time been the only steroids bearing an extra methyl group on their skeleton which have been identified in geological samples. They are presumably molecular fossils of $4 \alpha$-methylsterols occurring in widespread microscopic algae, the dinoflagellates 4 . However, steranes with an additional methyl group at position $2 \alpha$ or $3 \beta$ have recently been shown to occur in sediments of various geological ages 5 . We would now like to report the identification by synthesis of novel 2-methyl-, 3-methyl- and 6-methyltriaromatic steroids in marine shales and petroleums from the Paris basin.

The geological samples studied belong to the marine Jurassic formation (200 $\times 10^{6}$ years) of the Paris basin and are located at depths varying between $810 \mathrm{~m}$ and $2700 \mathrm{~m}$. Enriched triaromatic hydrocarbon fractions of sediment extracts and crude oils were separated by thin layer chromatography $\left(\mathrm{SiO}_{2}\right.$, hexane), based on the retention time of references (1-ethylnaphtalene, 1-methylphenanthrene, 1,2:5,6dibenzanthracene). Methyltriaromatic steroids are usually present in small amounts and require for their analysis the use of computerized gas chromatography-mass spectrometry (GC-MS) monitoring of the highly specific $\mathrm{m} / \mathrm{z}=245$ fragment ion which corresponds to the facile cleavage of the side chain at a benzylic position. The $\mathrm{m} / \mathrm{z}=245$ fragmentogram indeed showed that these samples contained, in addition to previously identified 4-methyl components $6,7\left(C_{21}, C_{22}, C_{27}-C_{29}\right.$ homologues essentially), several other isomers bearing a methyl group at a different position. The relative concentration of these isomers increased in more mature samples, implying their possible use as maturity parameters for geochemical studies. By analogy with changes previously cbserved in the phenanthrene series 8 the most likely substitution positions appeared to be 2,3 or 6 .<smiles>CC(=O)C1CCC2C3CC=C4C[C@@H](O)CCC4(C)C3CCC12C</smiles>

6<smiles>CC[C@]1(C)CCC2=C1C=Cc1cccc2c1</smiles>

2<smiles>CCC1CCC2C3CC=C4C[C@@H](O)CCC4(C)C3CCC12C</smiles>

$b, c, d, e, f, e$, $\mathrm{g}, \mathrm{h}, \mathrm{i}$<smiles></smiles>

3<smiles>[IH]=[Tl]</smiles>

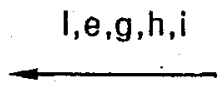<smiles>CCC(C)C1CCC2=CC(=O)CCC23CC1C3</smiles>

$k, f, e, g, h, i$<smiles>CC[C@]1(C)CCC2=C1C=CC1=C3C1=CC=c1ccc(C)cc1=C23</smiles>

1

Scheme 1. Reaction sequences used for the synthesis of triaromatic steroid hydrocarbons 1 , 2 and 3. a: Wolff-Kishner; b: MCPBA; c : MeMgl; d: Jones; e: $\mathrm{pTsOH}$; : $\mathrm{LiAlH}_{4}$; g: phenanthrenequinone; h: chloranil; i: $\mathrm{H}_{2}, \mathrm{Pd} / \mathrm{C}$; j: Oppenauer; k: (iPr-, cyclohexyl-) NLi, Mel, KOH; I: MeLi. 
1,2 and $3\left(\mathrm{C}_{21}\right)$ were synthesized based on a pathway previously described for the preparation of demethylated triaromatic steroids6,9, starting from pregnenolone 6 (scheme 1); 4 and 5 were obtained by

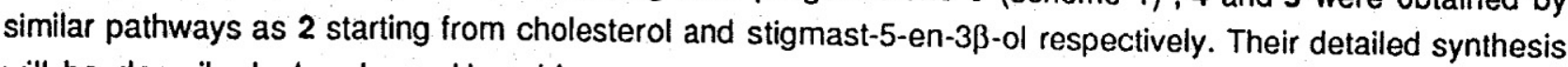
will be described elsewhere. Unambiguous structural assignments of intermediates were performed by extensive NMR experiments. The following NMR $\left(200 \mathrm{MHz}^{\mathrm{C}} \mathrm{CDCl}_{3}, \delta \mathrm{ppm}, \mathrm{J} \mathrm{Hz}\right)$ and mass spectral $(\mathrm{EI}, 70 \mathrm{eV})$ data were obtained:

1 : NMR : $0.86\left(\mathrm{t}, \mathrm{J}=7.4,3 \mathrm{H}, 21-\mathrm{CH}_{3}\right) ; 1.34\left(\mathrm{~s}, 3 \mathrm{H}, 18-\mathrm{CH}_{3}\right) ; 1.55-1.83\left(\mathrm{~m}, 2 \mathrm{H}, 20-\mathrm{CH}_{2}\right) ; 1.90-2.30(\mathrm{~m}, 2 \mathrm{H}, 16-$ $\mathrm{CH}_{2}$ ); 2.62 (s, 3H, CH 3 on $\left.\mathrm{C}-2\right) ; 3.26$ (t, J = 7.1-7.5, 2H, 15- $\mathrm{CH}_{2}$ ); 7.39 (dd, J3-4 = 8.1, J = 1.5, $1 \mathrm{H}, \mathrm{H}-3$ ); 7.41 (d, $J=8.5,1 H, H-12) ; 7,68$ (d; $J=9.7,1 H, H-6$ or $H-7) ; 7.73(d, J=9.5,1 H, H-7$ or $H-6) ; 7,77(d, J=8.2,1 H, H-4)$; $8.46(\mathrm{~s}, 1 \mathrm{H}, \mathrm{H}-1) ; 8.56(\mathrm{~d}, \mathrm{~J}=8.5,1 \mathrm{H}, \mathrm{H}-11) . \mathrm{MS}: \mathrm{m} / \mathrm{z}=274(\mathrm{M}+, 20 \%), 259\left(\mathrm{M}^{+}-\mathrm{CH}_{3}, 5 \%\right), 245\left(\mathrm{M}^{+}-\mathrm{C}_{2} \mathrm{H}_{5}\right.$, $100 \%), 230(12 \%), 229(13 \%), 215(13 \%), 115(7 \%), 101(4 \%)$.

2 : NMR : $0.87\left(\mathrm{t}, \mathrm{J}=7.4,3 \mathrm{H}, 21-\mathrm{CH}_{3}\right) ; 1.34\left(\mathrm{~s}, 3 \mathrm{H}, 18-\mathrm{CH}_{3}\right) ; 1.60-1.80\left(\mathrm{~m}, 2 \mathrm{H}, 20-\mathrm{CH}_{2}\right) ; 1.90-2.40(\mathrm{~m}, 2 \mathrm{H}, 16-$ $\mathrm{CH}_{2}$ ); $2.56\left(\mathrm{~s}, 3 \mathrm{H}, \mathrm{CH}_{3}\right.$ on $\left.\mathrm{C}-3\right) ; 3.27\left(\mathrm{t}, \mathrm{J}=7.3,2 \mathrm{H}, 15-\mathrm{CH}_{2}\right) ; 7.42(\mathrm{~d}, \mathrm{~J}=8.4,1 \mathrm{H}, \mathrm{H}-12) ; 7.46$ (dd, $\mathrm{J}_{1-2}=8.2, \mathrm{~J}$ $=1.6,1 \mathrm{H}, \mathrm{H}-2) ; 7.66(\mathrm{~s}, 1 \mathrm{H}, \mathrm{H}-4) ; 7.69(\mathrm{~d}, \mathrm{~J}=9.0,1 \mathrm{H}, \mathrm{H}-6$ or H-7); 7.75 (d, J=9.1, 1H, H-7 or H-6); 8.54 (d, J = 8.4, $1 \mathrm{H}, \mathrm{H}-11) ; 8.57(\mathrm{~d}, \mathrm{~J}=8.5,1 \mathrm{H}, \mathrm{H}-1)$. MS : $\mathrm{m} / \mathrm{z}=274\left(\mathrm{M}^{+}, 20 \%\right), 259\left(\mathrm{M}^{+}-\mathrm{CH}_{3}, 4 \%\right), 245\left(\mathrm{M}^{+}-\mathrm{C}_{2} \mathrm{H}_{5}\right.$. $100 \%), 230(12 \%), 229$ (17\%), $215(16 \%), 115(7 \%), 101(4 \%)$.

$3: \mathrm{NMR}: 0.86\left(\mathrm{t}, \mathrm{J}=7.4,3 \mathrm{H}, 21-\mathrm{CH}_{3}\right) ; 1.44\left(\mathrm{~s}, 3 \mathrm{H}, 18-\mathrm{CH}_{3}\right) ; 1.55-1.85\left(\mathrm{~m}, 2 \mathrm{H}, 20-\mathrm{CH}_{2}\right) ; 1.90-2.30(\mathrm{~m}, 2 \mathrm{H}, 16-$ $\mathrm{CH}_{2}$ ); 2.76 (s, 3H, $\mathrm{CH}_{3}$ on $\left.\mathrm{C}-6\right) ; 3.25$ (t, J = 7.3, 2H, 15- $\left.\mathrm{CH}_{2}\right) ; 7.37$ (d, J = 8.5, $\left.1 \mathrm{H}, \mathrm{H}-12\right) ; 7.60$ (s, $1 \mathrm{H}, \mathrm{H}-7$ ); 7.55-7.69 (m, $2 \mathrm{H}, \mathrm{H}-2$ and $\mathrm{H}-3) ; 8.00-8.08(\mathrm{~m}, 1 \mathrm{H}, \mathrm{H}-4) ; 8.54(\mathrm{~d}, \mathrm{~J}=8.4,1 \mathrm{H}, \mathrm{H}-11) ; 8.68-8.74(\mathrm{~m}, 1 \mathrm{H}, \mathrm{H}-1)$. MS $: \mathrm{m} / \mathrm{z}=274\left(\mathrm{M}^{+}, 20 \%\right), 259\left(\mathrm{M}^{+}-\mathrm{CH}_{3}, 5 \%\right), 245\left(\mathrm{M}^{+}-\mathrm{C}_{2} \mathrm{H}_{5}, 100 \%\right), 230(12 \%), 229(13 \%), 215(13 \%)$, $115(7 \%), 101(4 \%)$.

4 : NMR : $0.75\left(\mathrm{~d}, \mathrm{~J}=6.7,3 \mathrm{H}, 21-\mathrm{CH}_{3}\right) ; 0.88\left(\mathrm{~d}, \mathrm{~J}=6.5,6 \mathrm{H}, 26-\mathrm{CH}_{3}\right.$ and $\left.27-\mathrm{CH}_{3}\right) ; 1.34\left(\mathrm{~s}, 3 \mathrm{H}, 18-\mathrm{CH}_{3}\right) ; 1.70$ $2.40\left(\mathrm{~m}, 2 \mathrm{H}, 16-\mathrm{CH}_{2}\right) ; 2.56\left(\mathrm{~s}, 3 \mathrm{H}, \mathrm{CH}_{3}\right.$ on $\left.\mathrm{C}-3\right) ; 3.26\left(\mathrm{t}, \mathrm{J}=7.4,2 \mathrm{H}, 15-\mathrm{CH}_{2}\right) ; 7.41(\mathrm{~d}, \mathrm{~J}=8.6,1 \mathrm{H}, \mathrm{H}-12) ; 7.46$ (dd, $\left.\mathrm{J}_{1-2}=8-9, \mathrm{~J}=1.5,1 \mathrm{H}, \mathrm{H}-2\right) ; 7.66(\mathrm{~s}, 1 \mathrm{H}, \mathrm{H}-4) ; 7.68(\mathrm{~d}, \mathrm{~J}=8.6,1 \mathrm{H}, \mathrm{H}-6$ or $\mathrm{H}-7$ ); $7.74(\mathrm{~d}, \mathrm{~J}=9.2,1 \mathrm{H}, \mathrm{H}-7$ or $\mathrm{H}-6) ; 8.52(\mathrm{~d}, \mathrm{~J}=8.3,1 \mathrm{H}, \mathrm{H}-11) ; 8.57(\mathrm{~d}, \mathrm{~J}=8.4,1 \mathrm{H}, \mathrm{H}-1)$. MS : $\mathrm{m} / \mathrm{z}=358\left(\mathrm{M}^{+}, 4 \%\right), 245\left(\mathrm{M}^{+}-\mathrm{C}_{8} \mathrm{H}_{17}, 100\right.$ \%), $230(7 \%), 229(6 \%), 215(7 \%), 57(2 \%), 43(5 \%)$.

5 : NMR : $0.72\left(\mathrm{~d}, \mathrm{~J}=6.7,3 \mathrm{H}, 21-\mathrm{CH}_{3}\right) ; 0.814\left(\mathrm{~d}, \mathrm{~J}=6.7,3 \mathrm{H}, 26-\mathrm{CH}_{3}\right.$ or $\left.27-\mathrm{CH}_{3}\right) ; 0.847(\mathrm{~d}, \mathrm{~J}=6.7,3 \mathrm{H}, 26-$ $\mathrm{CH}_{3}$ or $\left.27-\mathrm{CH}_{3}\right) ; 0.847\left(\mathrm{t}, \mathrm{J}=7.3,3 \mathrm{H}, 29-\mathrm{CH}_{3}\right) ; 1.33\left(\mathrm{~s}, 3 \mathrm{H}, 18-\mathrm{CH}_{3}\right) ; 1.70-2.40\left(\mathrm{~m}, 2 \mathrm{H}, 16-\mathrm{CH}_{2}\right) ; 2.54(\mathrm{~s}, 3 \mathrm{H}$, $\mathrm{CH}_{3}$ on $\left.\mathrm{C}-3\right) ; 3.24$ (t, J = 7.4, 2H, 15- $\left.\mathrm{CH}_{2}\right) ; 7.39(\mathrm{~d}, \mathrm{~J}=8.6,1 \mathrm{H}, \mathrm{H}-12) ; 7.41-7.47(\mathrm{~m}, 1 \mathrm{H}, \mathrm{H}-2) ; 7.64(\mathrm{~s}, 1 \mathrm{H}, \mathrm{H}-4)$; $7.66(d, J=8.6,1 H, H-6$ or $H-7) ; 7.72(d, J=9.2,1 H, H-7$ or $H-6) ; 8.50(d, J=8.3,1 H, H-11) ; 8.55(d, J=8.4$, $1 \mathrm{H}, \mathrm{H}-1) \cdot \mathrm{MS}: \mathrm{m} / \mathrm{z}=286\left(\mathrm{M}^{+}, 4 \%\right), 245\left(\mathrm{M}^{+}-\mathrm{C}_{10} \mathrm{H}_{21}, 100 \%\right), 230(7 \%), 229(5 \%), 43(5 \%)$.

Compounds 1-5 were identified in HPLC fractions (RP-18, methanol) from geological samples by the following criteria : comparison of mass spectra and coelution, in single ion mass fragmentometry $(\mathrm{m} / \mathrm{z}=245)$, on two or three phases of various polarity in capillary column GC-MS (SE 30, DB 17, DB WAX). On DB 17 the elution order of the $\mathrm{C}_{21}$ compounds was $1,2,3 ; 2$-methyl , 3-methyl and 6-methyltriaromatic steroids $(1,2,3$, $4,5)$ elute before their 4 -methyl and 1-methyl isomers. On SE 30 compounds 1 and 2 nearly coelute. The configuration at $\mathrm{C}-24$ of the $\mathrm{C}_{28}$ and $\mathrm{C}_{29}$ geological homologues remains undetermined since the two diastereoisomers cannot be separated under typical gas chromatographic conditions. 
Several hypotheses can be formulated in order to explain the origin and formation of these novel triaromatic steroid hydrocarbons in the subsurface. One interesting point of information is that the relative concentrations of the 2-methyl and 3-methyl isomers increase with depth as compared with their 4-methyl counterparts. It is therefore likely that they could derive from the latter by a methyl shift leading to the more stable 2-methyl and 3-methyl components. This abundance change would be expected and is similar to observations made in the methylphenanthrene series 8 . The implied rearrangement could be catalysed by acid sites in the sediment matrix, in particular those occurring in clay minerals. On the other hand, one cannot exclude that the 2-methyl- and 3-methyltriaromatic steroids may arise from the same precursors as the recently characterized $2 \alpha$-and $3 \beta$-methylsteranes 5 , and could become concentrated in more mature geological samples due to the preferential degradation of the less stable 4-methyl isomers.

6-methyltriaromatic steroids could also be formed by rearrangement of their 4-methyl counterparts (they would be expected to have a similar stability since they show almost identical periplanar interactions) although this process would be energetically less favoured than that above. Alternatively, and more likely, they could arise during the further aromatization of rearranged ring $\mathrm{C}$ monoaromatic steroid hydrocarbons 10 by a methyl shift from $\mathrm{C}-5$ to $\mathrm{C}-6$. Finally, their origin from uncommon 6-methyl ring B monoaromatic sterols recently observed in a soil amoeba11 cannot be excluded.

Whatever the origin and mode of formation of these new compounds, our studies on the sedimentary series of the Paris basin have shown that they can be useful as molecular maturity parameters for geochemical purposes.

Acknowledgments: this research was supported by the Institut Français du Pétrole and the Centre National de la Recherche Scientifique (C.N.R.S.). We are grateful to E.Krempp, P. Wehrung and G.Teller for the NMR and mass spectral measurements.

\section{References:}

1 for a review see MACKENZIE A.S., BRASSELL S.C., EGLINTON G., MAXWELL J.R., Science, 217, 491504 (1982).

2 RUBINSTEIN I., ALBRECHT P., J. Chem. Soc., Chem. Commun., 957-958 (1975).

3 ENSMINGER A., JOLY G., ALBRECHT P., Tetrahedron Lett., 18, 1575-1578 (1978).

4 ROBINSON N., EGLINTON G., BRASSEL S.C., CRANWELL P.A., Nature, 308, 439-442 (1984).

5 SUMMONS R.E., CAPON R.J., Geochim. Cosmochim. Acta, 52, 2733-2736 (1988).

6 LUDWIG B., HUSSLER G., WEHRUNG P., ALBRECHT P., Tetrahedron Lett., 22, 3313-3316 (1981).

7 RIOLO J., Thèse de Doctorat, Université Louis Pasteur, Strasbourg, (1985).

8 RADKE M., WELTE D.H., in Advances in Organic Geochemistry 1981, M. BJOROY et al. eds, Wiley, New York, $p$ 504-512.

9 DANNENBERG H., NEUMANN H.G., Liebigs Ann. Chem., 675, 109-125 (1964).

10 RIOLO J., ALBRECHT P., Tetrahedron Lett., 26, 2701-2704 (1985).

11 BISSERET P., ADAM H., ROHMER M., J. Chem. Soc., Chem. Commun., 693-695 (1987). 